\title{
Estimativas de repetibilidade para produção de matéria seca em alfafa
}

\author{
Estimates of repeatability for alfalfa dry matter production
}

\author{
Fausto de Souza-Sobrinho ${ }^{1}$ Francisco José da Silva Lédo ${ }^{1}$ Antônio Vander Pereira ${ }^{1}$ \\ Milton Andrade Botrel ${ }^{2}$ Antônio Ricardo Evangelista ${ }^{3}$ Maria Celuta Machado Viana ${ }^{4}$
}

\section{RESUMO}

Com o aumento da competitividade do setor produtivo, a pecuária leiteira brasileira busca, de todas as formas, sistemas de produção que obtenham maior produtividade e menor custo. Entre as estratégias adotadas para tal fim, estão a melhoria da qualidade do rebanho e da forragem fornecida aos animais, de modo que estes expressem todo o seu potencial produtivo. A alfafa, conhecida pela excelente qualidade forrageira $e$ boa palatabilidade, surge como boa opção. Entretanto, ainda há muito o que se trabalhar para a obtenção de cultivares melhor adaptadas às condições tropicais. Informações teóricas sobre características de importância forrageira são necessárias para a orientação dos programas de melhoramento. Nesse sentido, o objetivo deste trabalho foi estimar o coeficiente de repetibilidade para a produtividade de matéria seca de alfafa na região Sudeste do Brasil e determinar o número ideal de avaliações para a seleção dos genótipos superiores, por meio de quatro metodologias diferentes. Utilizaram-se dados de produtividade de matéria seca obtidos em quatro ensaios de comparação de cultivares de alfafa, conduzidos em diferentes regiões do Estado de Minas Gerais. Estimouse o coeficiente de repetibilidade (r), para cada um dos ensaios, por meio dos métodos da análise de variância (Anova), dos componentes principais com base na matriz de covariância (CP-1) e de correlação (CP-2), e pela análise estrutural baseada na matriz de correlação. Observou-se que houve boa concordância entre as diferentes metodologias de estimação do coeficiente de repetibilidade em todos os ensaios. A estimativa média de repetibilidade para produção de matéria seca de alfafa nos quatro ensaios avaliados foi de 0,59, com coeficiente de determinação médio de 0,96. Constatou-se, também, que a realização de apenas quatro cortes, em média, foram suficientes para se conhecer o real valor genotípico das cultivares testadas, com $85 \%$ de confiabilidade.

Palavras-chave: Medicago sativa L., produtividade, forrageira, melhoramento.

\section{ABSTRACT}

Along with the increasing competition in its primary production area the Brazilian dairy searchs for production systems that result higher productivity and lower costs. Among the adopted strategies are the herd and forage improvement. Alfalfa, known for its excellent nutritional quality and palatability, becomes an option. However, to obtain cultivars well adapted to tropical conditions still requires a lot of time and work. Theoretical information regarding forage value characters are required in order to orient improvement programs. So, the objective of this work was to estimate the repeatability coefficient of alfalfa dry matter productivity in Brazil southeast region and to determinate the ideal evaluation number necessary to select higher genotypes. Four methodologies were used. Dry matter productivity data from four alfalfa evaluation experiments carried in different areas of Minas Gerais State were used. The repeatability coefficient (r) for each experiment was estimated using variance analysis (Anova) of the main components based on the covariance $(C P-1)$ and the correlation (CP-2) matrix and by the structural analysis based on the correlation matrix. A good response among the four methodologies was observed in all experiments. The average estimate for repeatability of alfalfa dry matter production for all experiments was 0,59, with the determination coefficient average of 0,96. What was also noted was that four cuts, in average, were sufficient to shown with $85 \%$ confidence the real genotipic value of the evaluated cultivars.

Key words: Medicago sativa L., yield, breeding, forage.

\footnotetext{
${ }^{1}$ Engenheiro Agônomo, Doutor, Embrapa Gado de Leite. Rua Eugênio do Nascimento, 610, 36038-330, Juiz de Fora, MG. E-mail: fausto@cnpgl.embrapa.br

${ }^{2}$ Engenheiro Agrônomo, Mestre, Embrapa Gado de Leite.

${ }^{3}$ Engenheiro Agrônomo, Doutor, Professor Titular, Universidade Federal de Lavras, Departamento de Zootecnia, CP 37, 37200000, Lavras, MG.

${ }^{4}$ Engenheiro Agrônomo, Mestre, EPAMIG, 35701-970, Sete Lagoas, MG.
} 


\section{INTRODUÇÃO}

As condições atuais do mercado, com a maior disponibilidade de informações e a globalização, levaram à maior competitividade em todos os setores produtivos. A pecuária leiteira se viu forçada a adotar sistemas de intensificação da produção que possibilitem a obtenção de maior produtividade e menor custo. A implantação desse novo sistema produtivo normalmente envolve a utilização de animais de alta capacidade produtiva e de alimentos volumosos de alta qualidade que permitam a expressão de todo o potencial genético animal para a produção de leite (FERREIRA \& PEREIRA, 1999).

Dentre as diversas espécies forrageiras cultivadas no Brasil, a alfafa (Medicago sativa $L$.) se destaca principalmente pela alta produtividade, qualidade da forragem e aceitação pelos animais. Em países de clima temperado, como EUA e Argentina, é o volumoso mais utilizado na alimentação de vacas de leite (CASTILLO \& GALLARDO, 1995), podendo ser oferecido aos animais como feno, silagem, verde picado e sob a forma de pastejo, conseguindo-se excelentes resultados em termos de produção de leite. Por se tratar de uma espécie de origem de clima temperado, as cultivares disponíveis no mercado normalmente apresentam problemas de adaptação ao ambiente tropicial, principalmente em relação à susceptibilidade a pragas e doenças. Isto certamente tem contribuído na pequena expansão das áreas cultivadas com esta leguminosa nas condições tropicais brasileiras. No entanto, considerando a existência de variabilidade genética no germoplasma de alfafa para a maioria das características de importância forrageira, torna-se possível a seleção de novos materiais genéticos melhor adaptados às condições tropicais (PEREIRA et al., 2001).

Dentre as características agronômicas importantes para o melhoramento de forrageiras, a produtividade de matéria seca se destaca e tem sido avaliada na maioria dos ensaios comparativos de desempenho de cultivares de alfafa (JULIER et al., 2000; BOTREL et al., 2001; GUINES et al., 2002; KATEPAMUPONDWA et al., 2002). A produtividade é o resultado final de diversas outras características, como resistência a pragas e doenças, tolerância aos diversos tipos de estresses abióticos, podendo ser utilizada como característica representativa da adaptação dos diferentes materiais genéticos. Este fato torna-se importante por facilitar a avaliação dos ensaios, pelo menor número de características avaliadas e reduzir o custo final dos trabalhos de melhoramento.
Uma das dificuldades encontradas para o melhoramento de forrageiras diz respeito à determinação do número de avaliações necessárias para estimar as diferenças entre materiais genotípicos. No caso da alfafa, em que são realizados vários cortes na mesma área, o melhoramento deve considerar os dados de diversas avaliações antes da seleção dos genótipos mais promissores. Essas avaliações sucessivas permitem a estimativa do coeficiente de repetibilidade das características avaliadas, ou seja, permitem saber qual a probabilidade daquele resultado se repetir em avaliações futuras. Determina-se, também, o número de avaliações necessárias para se predizer, com confiabilidade, o comportamento dos materiais avaliados. A obtenção de estimativas elevadas de repetibilidade para determinado caráter indica que é possível predizer o valor real do indivíduo utilizando um número relativamente pequeno de medições (CRUZ \& REGAZZI, 1997). Com isso, há possibilidade de redução no número de avaliações e do tempo exigido para a seleção dos genótipos promissores, contribuindo substancialmente para a orientação dos programas de melhoramento.

Alguns estudos de repetibilidade em alfafa, baseados em dados obtidos no Brasil, encontram-se disponíveis na literatura (PEREIRA et al., 1998; FERREIRA et al., 1999; BOTREL et al. 2000). Entretanto, a maioria desses estudos foram realizados separando os cortes de avaliação em dois períodos, águas e seca, obtendo-se estimativas de repetibilidade para cada um deles. Como normalmente a alfafa é cultivada realizando irrigações complementares no período seco e há pouco interesse em recomendar uma cultivar para um período específico do ano, as estimativas de repetibilidade obtidas por esses estudos são de pouca utilidade. Na avaliação e seleção de genótipos de alfafa, o interesse está em identificar os materiais com alta produtividade e que apresentem maior estabilidade de produção ao longo do ano. Assim, os estudos de repetibilidade devem considerar todos os cortes realizados sucessivamente ao longo do tempo.

O objetivo deste trabalho foi estimar a repetibilidade para produtividade de matéria seca, sob condições de ambiente tropical, e determinar o número ideal de avaliações para a seleção dos genótipos superiores, por meio de quatro metodologias diferentes.

\section{MATERIAL E MÉTODOS}

As estimativas de repetibilidade foram obtidas a partir dos dados de quatro ensaios de 
comparação de cultivares de alfafa, sendo dois deles conduzidos no Campo Experimental de Coronel Pacheco-MG, da Embrapa Gado de Leite (ensaios CO e CO1), um na Universidade Federal de Lavras (UFLA - Lavras/MG) (ensaio LA), e o último, no Campo Experimental da Epamig, em Sete Lagoas-MG (ensaio SL).

Em um dos ensaios de Coronel Pacheco (CO-1) avaliaram-se 59 cultivares de alfafa, constituídas, em sua maioria, de introduções de outros países, realizadas pela Embrapa Gado de Leite. O delineamento utilizado foi de blocos ao acaso com duas repetições, e parcelas de três linhas de $4 \mathrm{~m}$ de comprimento, espaçadas de $0,3 \mathrm{~m}$ entre elas, considerando-se como área útil $1,2 \mathrm{~m}^{2}$ da fileira central. Foram realizados 16 cortes de avaliação.

Os outros três ensaios faziam parte da Rede Nacional de Avaliação de Cultivares de Alfafa (RENACAL), coordenada pela Embrapa Gado de Leite, e foram estabelecidos e conduzidos segundo uma metodologia padrão. Embora o número de cultivares e de cortes realizados não tenham sido os mesmos, havia 28 cultivares comuns aos três ensaios. As cultivares avaliadas foram pré-selecionadas pela Embrapa Gado de Leite, a partir de introduções de outros países. O delineamento utilizado foi de blocos ao acaso com três repetições e parcelas de cinco linhas de $5 \mathrm{~m}$ de comprimento, espaçadas de $0,2 \mathrm{~m}$ uma da outra. A parcela útil foi constituída pelas três linhas centrais, descartandose $0,5 \mathrm{~m}$ de cada extremidade, ou seja, $2,4 \mathrm{~m}^{2}$ de área útil. Em Coronel Pacheco foram avaliadas 30 cultivares em 20 cortes sucessivos, em Lavras 35 cultivares e 15 cortes e em Sete Lagoas 28 cultivares e 28 cortes.

Em todos os ensaios empregou-se uma densidade de semeadura de $20 \mathrm{~kg}$ de sementes por hectare. As sementes foram previamente inoculadas com Rhizobium melilotii BR 7407 proveniente da Embrapa Agrobiologia e peletizadas com calcário dolomítico. A adubação de plantio, assim como a correção do solo e a irrigação, foram realizadas com base nos resultados das análises de solo e das recomendações para a cultura (COMISSÃO..., 1999).

Após o plantio, quando a maioria das cultivares atingiram o pleno florescimento procedeuse um corte de uniformização a uma altura de $5 \mathrm{~cm}$ acima do solo em todas as parcelas. A partir de então foram realizados cortes sucessivos sempre que $50 \%$ das parcelas apresentassem pelo menos $10 \%$ de florescimento. Em cada um dos cortes, avaliou-se a produção de matéria seca de forragem (MS), em kg/ha. Para tanto, anotaram-se os dados referentes ao peso verde das parcelas e retirou-se uma amostra para a determinação do teor de matéria seca.
Inicialmente os dados obtidos em cada ensaio foram submetidos à análise de variância considerando os tratamentos dispostos no esquema de parcela subdividida no tempo, sendo as parcelas representadas pelas cultivares e as subparcelas pelos cortes (RAMALHO et al., 2000a).

No estudo da repetibilidade, foi considerado o modelo reduzido (CRUZ \& REGAZZI, 1997): em que: $Y_{i j}=\mu+G_{i}+C_{j}+\varepsilon_{i j}$

$\mathrm{Y}_{\mathrm{ij}}=$ média da i-ésima variedade no j-ésimo corte;

$\mu=$ média geral do ensaio;

$\mathrm{G}_{\mathrm{i}}=$ efeito da i-ésima variedade confundido com as influências permanentes do ambiente (corte);

$\mathrm{C}_{\mathrm{j}}=$ efeito do j-ésimo corte;

$\varepsilon_{\mathrm{ij}}=$ efeito aleatório que envolve outras causas de variação não incluídas no modelo.

Para cada um dos ensaios estudados, estimou-se o coeficiente de repetibilidade (r) por meio dos métodos da análise de variância (Anova), dos componentes principais com base na matriz de covariância (CP-1) e de correlação (CP-2), e pela análise estrutural (AE) baseada na matriz de correlação (CRUZ \& REGAZZI, 1997).

\section{RESULTADOS E DISCUSSÃO}

Inicialmente ressalta-se a boa precisão experimental, medida por meio dos coeficientes de variação, obtida em todos os ensaios realizados. Os coeficientes de variação das análises individuais oscilaram de 25,1\%, em Lavras, a 13,8\% em Sete Lagoas. Esses resultados contribuem para aumentar a confiabilidade das estimativas e dos resultados obtidos neste trabalho.

Em todos os locais avaliados observaram-se diferenças significativas para as fontes de variação cultivares e cortes, indicando que as cultivares de alfafa testadas apresentam diferenças para a produção de matéria seca na Região Sudeste do Brasil e que esta produção é afetada pelos cortes sucessivos. A interação cultivares $x$ cortes também foi significativa em todos os ensaios, evidenciando que o comportamento das cultivares não foi consistente nos diferentes cortes realizados, ou seja, a ordem de classificação de produtividade das cultivares foi alterada nos sucessivos cortes. Esses resultados concordam com a maioria dos trabalhos encontrados na literatura, e evidenciam ocorrência de comportamento variável entre cultivares sob diferentes épocas de corte (OLIVEIRA et al., 1993; PEREIRA et al., 1998; FERREIRA et al., 1999; BOTREL et al., 2000). 
Como o comportamento das cultivares não é constante nas sucessivas avaliações de produtividade de matéria seca, necessita-se de maior número de cortes para a confirmação do verdadeiro potencial produtivo dos diferentes genótipos. Entretanto, a realização de avaliações (cortes) sucessivas, além de encarecerem os ensaios, retardam a obtenção de resultados que permitam a seleção dos materiais mais promissores. Nesse sentido, a estimativa do coeficiente de repetibilidade pode ser bastante útil por fornecer informações a respeito da confiabilidade dos resultados gerados pelos ensaios. Além disso essa estimativa permite inferir sobre o número mínimo de cortes necessários para se conhecer o real valor genotípico dos materiais avaliados (CRUZ \& REGAZZI, 1997).

As estimativas dos coeficientes de repetibilidade (Tabela 1) para produção de matéria seca obtidas nos três ensaios da RENACAL (CO-2, LA e $\mathrm{SL}$ ), foram concordantes entre si, e sempre superiores a 0,58, com média de 0,64 . Segundo CRUZ \& REGAZZI (1997), a repetibilidade varia em função da natureza do caráter, com as propriedades genéticas da população e com as condições ambientais sob as quais os indivíduos são mantidos. Como os ensaios foram realizados na região Sudeste, utilizando uma metodologia padronizada, onde mais de $90 \%$ dos genótipos avaliados eram comuns aos três ensaios, essa concordância nas estimativas de repetibilidade já era esperada. Também, se considerarmos que o número de cortes de avaliação realizados nos ensaios foram de 15,20 e 28 cortes, respectivamente para LA, CO-2 e SL, há uma indicação de que o aumento do número de medições não teve grande influência na magnitude das estimativas de repetibilidade obtidas.

Por se tratar de uma característica quantitativa, controlada por vários locos e com efeito ambiental pronunciado (RAMALHO et al., 2000b;
RAMALHO et al., 2001), a magnitude destas estimativas podem ser consideradas elevadas, e indicam confiabilidade na identificação dos melhores genótipos, com base nas médias obtidas. Além disso, os coeficientes de determinação $\left(\mathrm{R}^{2}\right)$ associados às estimativas dos coeficientes de repetibilidade foram elevados, variando de 0,93 a 0,98, com média de 0,97. Assim, a identificação das melhores cultivares pode ser realizada com $93 \%$ a $98 \%$ de confiabilidade de se estar inferindo sobre o valor real dos genótipos. Dessa forma, os resultados obtidos nos ensaios da RENACAL foram bastante confiáveis, sugerindo que a seleção dos melhores genótipos para a produtividade de matéria seca de forragem em alfafa pode ser realizada com sucesso.

Para o ensaio CO-1, as estimativas dos coeficientes de repetibilidade foram inferiores às obtidas nos ensaios da RENACAL, apresentando média de 0,45 . O coeficiente de determinação variou de 0,92 a 0,93 , indicando que os 16 cortes realizados permitiram uma predição do valor real dos genótipos acima de $92 \%$, o que pode ser considerado satisfatório. A maioria dos genótipos avaliados nos quatro ensaios, foram provenientes de introduções de outros países realizadas pela Embrapa Gado de Leite; entretanto, nos ensaios da RENACAL os genótipos utilizados foram pré-selecionados quanto a sua maior chance de adaptação ao clima tropical. Já no ensaio CO-1 foi avaliado um número bem maior de genótipos (59) que não passaram por nenhum processo de pré-seleção. Genótipos menos adaptados ao clima tropical são mais susceptíveis a ataques de pragas e doenças, e apresentam baixa persistência, o que pode justificar a menor magnitude das estimativas de repetibilidade obtidas no ensaio de CO-1.

Constatou-se também, que as estimativas do coeficiente de repetibilidade obtidas em todos os

Tabela 1 - Estimativas de repetibilidade (r) e do coeficiente de determinação genotípico $\left(\mathrm{R}^{2}\right)$ obtidos da análise da produção de matéria seca avaliada em cultivares de alfafa, baseada em diferentes metodologias

\begin{tabular}{|c|c|c|c|c|c|c|c|c|}
\hline \multirow{2}{*}{ Método } & \multicolumn{2}{|c|}{ CO-1 } & \multicolumn{2}{|c|}{ CO-2 } & \multicolumn{2}{|c|}{ LA } & \multicolumn{2}{|c|}{ SL } \\
\hline & $\mathbf{r}$ & $\mathbf{R}^{2}$ & $\mathbf{r}$ & $\mathbf{R}^{2}$ & $\mathbf{r}$ & $\mathbf{R}^{2}$ & $\mathbf{r}$ & $\mathbf{R}^{2}$ \\
\hline Anova & 0,42 & 0,92 & 0,60 & 0,97 & 0,64 & 0,96 & 0,58 & 0,97 \\
\hline CP (covariância) & 0,45 & 0,93 & 0,66 & 0,97 & 0,68 & 0,93 & 0,62 & 0,98 \\
\hline CP (correlação) & 0,46 & 0,93 & 0,72 & 0,98 & 0,66 & 0,97 & 0,61 & 0,98 \\
\hline AE (correlação) & 0,45 & 0,93 & 0,77 & 0,98 & 0,66 & 0,97 & 0,60 & 0,98 \\
\hline
\end{tabular}

CO-1 - Coronel Pacheco 60 cultivares.

CO-2 - Coronel Pacheco Renacal.

LA - Lavras (MG)

SL - Sete Lagoas (MG) 
ensaios, pelas diferentes metodologias, foram concordantes entre si. A maior discrepância foi observada no ensaio 2 de Coronel Pacheco (CO-2) entre a estimativa obtida por meio da análise de variância $(\mathrm{r}=0,60)$ e aquela obtida pela análise estrutural (AE) baseada na matriz de correlação $(r=0,77)$. Essa coincidência entre as metodologias empregadas para estimativa de repetibilidade da produção de matéria seca em alfafa concorda com estudos anteriores (PEREIRA et al., 1998; BOTREL et al., 2000) e indica a possibilidade de utilização de apenas uma das metodologias apresentadas, facilitando a interpretação dos resultados e difundindo o seu emprego em novos estudos.

Considerando-se todos os dados de todos os experimentos, as estimativas de repetibilidade variaram de 0,42 a 0,77 , concordando com os relatados na literatura em outros trabalhos de repetibilidade em alfafa (PEREIRA et al., 1998; FERREIRA et al.,1999; BOTREL et al., 2000). Entretanto, nos trabalhos de FERREIRA et al. (1999) e BOTREL et al. (2000), os estudos de repetibilidade foram realizados em dois períodos distintos, ou seja, fez-se um estudo para a repetibilidade nos cortes do período das águas e outro nos da seca, o que prejudica a comparação desses resultados com os obtidos neste trabalho. No entanto, quando se faz a média dos coeficientes encontrados nos dois períodos, a magnitude da repetibilidade para a produção de matéria seca mostrou-se semelhante àquela observada neste trabalho, próxima de 0,60.

$\mathrm{O}$ número de medições necessárias, com diferentes coeficientes de determinação genotípica, considerando as diferentes metodologias de estimação e locais, estão apresentados na tabela 2. Constatouse, à semelhança da repetibilidade, que o número de cortes exigidos em CO-1 foi, em média, o dobro do necessário nos ensaios da RENACAL, para atingir os mesmos valores de $\mathrm{R}^{2}$.

De modo geral, o número de medições necessárias para se determinar o valor genotípico dos materiais de alfafa avaliados foi bastante baixo. Considerando-se um $\mathrm{R}^{2}$ de 0,85 , o número de cortes variou de 7,7 em CO-1, a 2,3 em CO-2 (Tabela 2). Os dados médios, envolvendo os quatro locais e as diferentes metodologias de estimação, indicaram que aproximadamente 4 cortes são suficientes, com $85 \%$ de confiabilidade, para a seleção dos melhores genótipos.

Esses resultados demonstram que não é necessária a realização de grande número de cortes nos ensaios de alfafa para a seleção dos genótipos mais promissores. Com a possibilidade de se realizar a seleção baseada apenas em quatro avaliações nos estágios iniciais dos programas de melhoramento, onde o número de genótipos avaliados normalmente é grande, haverá uma grande redução no tempo gasto em cada ciclo de seleção melhorando a eficiência dos programas. Parte dos recursos economizados com a diminuição no número de avaliações realizadas poderá, ainda, ser investido no aumento da precisão experimental dos ensaios, por meio da utilização de maior número de repetições e de parcelas maiores, melhorando ainda mais a qualidade e confiabilidade dos resultados obtidos. Com isso algumas etapas dos programas de melhoramento se tornam mais ágeis, contribuindo para um processo mais dinâmico e aumentando as chances de sucesso.

Entretanto, em estágios mais avançados o número de avaliações realizadas deve ser maior, para se verificar não só o comportamento produtivo dos materiais mas também a sua estabilidade de produção ou persistência. Nessa fase, os genótipos encontramse em avaliação em rede para possível lançamento ou recomendação, exigindo maior confiabilidade na predição do valor real dos melhores genótipos. Nesse caso, baseando-se nos resultados obtidos nos ensaios da RENACAL, e um $\mathrm{R}^{2}$ de 0,95 , são necessários pelo menos 12 cortes de avaliação.

Vale lembrar que as fontes de variação cortes e a interação cultivares $\mathrm{x}$ cortes foram significativas, indicando que as avaliações sucessivas diferem entre si e que o comportamento dos genótipos não é totalmente consistente ao longo do tempo. Embora as estimativas de repetibilidade para produtividade de matéria seca tenham sido altas, sempre há, dentre os materiais selecionados, alguns que não confirmam o seu desempenho produtivo quando avaliados por um período maior de tempo. Isso é perfeitamente normal e, no caso específico da alfafa, já era esperado, visto que a maioria das cultivares estudadas foram introduzidas recentemente e não são adaptadas às condições edafoclimáticais brasileiras, apresentando variabilidade não só de comportamento produtivo, mas também de persistência. Ressalta-se, porém, que a etapa final de qualquer programa de melhoramento requer avaliações mais minuciosas para se ter maior confiança para a recomendação das cultivares a serem comercializadas.

Constata-se, portanto, que o número de avaliações necessárias para a realização da seleção dos genótipos de alfafa promissores não é grande. Especialmente nas etapas iniciais dos programas de melhoramento, a redução do número de cortes exigidos proporcionará redução no tempo gasto para a realização dos ciclos de seleção, aumentando a dinâmica e a eficiência dos mesmos. 
Tabela 2 - Números de medições necessárias para diferentes níveis de determinação genotípica $\left(\mathrm{R}^{2}\right)$, destinada à avaliação da produção de matéria seca em cultivares de alfafa

\begin{tabular}{|c|c|c|c|c|c|}
\hline \multirow{2}{*}{ Locais } & \multirow{2}{*}{$\mathrm{R}^{2}$} & \multicolumn{4}{|c|}{ Métodos } \\
\hline & & Anova (AV) & CP (covariância) & CP (correlação) & $\mathrm{AE}$ (correlação) \\
\hline \multirow{5}{*}{$\mathrm{CO}-1$} & 0,80 & 5,5 & 4,9 & 4,6 & 4,9 \\
\hline & 0,85 & 7,7 & 6,9 & 6,5 & 7,0 \\
\hline & 0,90 & 12,3 & 10,9 & 10,3 & 11,1 \\
\hline & 0,95 & 25,9 & 23,1 & 21,8 & 23,5 \\
\hline & 0,99 & 134,9 & 120,4 & 113,9 & 122,3 \\
\hline \multirow{5}{*}{$\mathrm{CO}-2$} & 0,80 & 2,6 & 2,1 & 1,6 & 1,7 \\
\hline & 0,85 & 3,7 & 2,9 & 2,3 & 2,5 \\
\hline & 0,90 & 5,9 & 4,6 & 3,5 & 3,9 \\
\hline & 0,95 & 12,5 & 9,7 & 7,5 & 8,3 \\
\hline & 0,99 & 64,9 & 50,7 & 38,9 & 43,2 \\
\hline \multirow{5}{*}{ LA } & 0,80 & 2,2 & 1,9 & 2,0 & 2,1 \\
\hline & 0,85 & 3,1 & 2,7 & 2,8 & 2,9 \\
\hline & 0,90 & 4,9 & 4,3 & 4,5 & 4,7 \\
\hline & 0,95 & 10,4 & 9,0 & 9,6 & 9,9 \\
\hline & 0,99 & 54,5 & 47,0 & 50,1 & 51,8 \\
\hline \multirow{5}{*}{ SL } & 0,80 & 2,9 & 2,5 & 2,5 & 2,7 \\
\hline & 0,85 & 4,1 & 3,5 & 3,6 & 3,8 \\
\hline & 0,90 & 6,5 & 5,6 & 5,7 & 6,1 \\
\hline & 0,95 & 13,8 & 11,8 & 12,1 & 12,8 \\
\hline & 0,99 & 72,9 & 61,5 & 62,8 & 67,0 \\
\hline
\end{tabular}

CO-1 - Coronel Pacheco 59 cultivares.

CO-2 - Coronel Pacheco Renacal.

LA - Lavras (MG)

$\mathrm{SL}-$ Sete Lagoas (MG)

Por tudo que foi mencionado, a determinação do número de avaliações necessárias para a realização da seleção com boa precisão ou confiabilidade merece atenção dos pesquisadores. Novos estudos devem ser conduzidos visando à melhoria do controle ambiental dos ensaios, para a maximização das estimativas de repetibilidade, e também para se determinar quantos cortes ou qual o período de tempo o material deve ser avaliado até a realização da seleção, tanto em etapas iniciais como no final dos programas de melhoramento.

\section{CONCLUSÕES}

Houve boa concordância entre as diferentes metodologias de estimação do coeficiente de repetibilidade em todos os ensaios, sendo obtido o valor médio de 0,59 .

A realização de quatro cortes foi suficiente para a estimativa do real valor genotípico das cultivares de alfafa, com $85 \%$ de confiabilidade.

\section{REFERÊNCIAS BIBLIOGRÁFICAS}

BOTREL, M.A. et al. Cultivares de alfafa em área de influência da Mata Atlântica no Estado de Minas Gerais. Pesquisa Agropecuária Brasileira, v.36, n.11, p.1437-1442, 2001.

BOTREL, M.A. et al. Estimativas de coeficientes de repetibilidade para produção de matéria seca em cultivares de alfafa, sob diferentes ambientes. Revista Ceres, v.47, n.274, p.651-663, 2000 .

CASTILLO, R.A.; GALlARDO, R.M. Suplementación de vacas lecheras en pastoreo de alfafa, concentrados e forrajes conservados. In: HIJANO, E.J.; NAVARRO, A. (Eds). La alfafa en la Argentina. San Juan : INTA, 1995. p.195204.

COMISSÃO DE FERTILIDADE DO SOLO DO ESTADO DE MINAS GERAIS. Recomendações para o uso de corretivos e fertilizantes em Minas Gerais - $5^{\text {a }}$ aproximação. RIBEIRO, A.C.; GUIMARÃES, P.T.G.; ALVARES, V.H.V. (Eds). Viçosa, MG, 1999. 359p.

CRUZ, C.D.; REGAZZI, A.J. Modelos biométricos aplicados ao melhoramento genético. 2 ed. Viçosa : UFV, 1997. 390p.

FERREIRA, R.P. et al. Avaliação de cultivares de alfafa e 
estimativas de repetibilidade de caracteres forrageiros. Pesquisa Agropecuária Brasileira, v.34, n.6, p.995-1002, 1999.

FERREIRA, R.P.; PEREIRA, A.V. Melhoramento de forrageiras. In: BORÉM, A. Melhoramento de espécies cultivadas. Viçosa : UFV, 1999. 817p.

GUINES, F. et al. Genetic control of quality traits of lucerne (Medicago sativa L.). Australian Journal of Agricultural Research, v.53, p.401-407, 2002.

JULIER, B.; HUYGHE, C.; ECALE, C. Within and among cultivar genetic variation in alfafa: Forage quality, morphology in a yield. Crop Science, v.40, p.365-369, 2000.

KATEPA-MUPONDWA, F.M.; CHRISTIE, B.R.; MICHAELS, T.E. An improved breedings strategy for autotetraploidy alfafa (Medicago sativa L.). Euphytica, v.123, p.139-146, 2002.

OLIVEIRA, P.R.D.; PAIM, N.R.; CZERMAINSKJ, A.B.C. Seleção para rendimento e qualidade de forragem em alfafa
Crioula. Pesquisa Agropecuária Brasileira, v.28, n.9, p.1039-1044, 1993.

PEREIRA, A.V. et al. Comportamento da alfafa cv. Crioula de diferentes origens e estimativas dos coeficientes de repetibilidade para caracteres forrageiros. Revista Brasileira de Zootecnia, v.27, n.4, p.686-690, 1998.

PEREIRA, A.V. et al. Melhoramento de forrageiras tropicais. In: NASS, L.L. et al. Recursos genéticos e melhoramento - Plantas. Rondonópolis : Fundação MT, 2001. 1183p.

RAMALHO, M.A.P.; ABREU, A.F.B.; SANTOS, J.B Melhoramento de espécies autógamas. In: In: NASS, L.L. et al. Recursos genéticos e melhoramento - Plantas. Rondonópolis : Fundação MT, 2001. 1183p.

RAMALHO, M.A.P.; FERREIRA, D.F.; OLIVEIRA, A.C. Experimentação em genética e melhoramento de plantas. Lavras : UFLA, 2000a. 326p.

RAMALHO, M.A.P.; SANTOS, J.B.; PINTO, C.A.B.P. Genética na agropecuária. Lavras : UFLA, 2000b. 472p. 